Applied Mathematical Sciences, Vol. 13, 2019, no. 11, 511 - 520

HIKARI Ltd, www.m-hikari.com

https://doi.org/10.12988/ams.2019.9463

\title{
A Global Stability Theory of Nonsmooth Periodic Orbits: Example I
}

\author{
Pascal Stiefenhofer ${ }^{1}$ \\ Brighton Business School, University of Brighton, UK \\ \& \\ Department of Mathematics, University of Sussex, UK \\ Peter Giesl \\ Department of Mathematics, University of Sussex, UK
}

This article is distributed under the Creative Commons by-nc-nd Attribution License. Copyright (c) 2019 Hikari Ltd.

\begin{abstract}
This paper considers a system of ordinary differential equations with discontinuous right-hand side. Such systems appear in economics in the context of economic growth. The paper first shows, at the case of a planar system, the derivation of a nonsmooth periodic orbit. It is then shown, by defining a Poincaré map, that this solution is globally stable. The limitations of this global stability theory are investigated and suggestions towards a local theory of stability are introduced.
\end{abstract}

Keywords: Dynamical Systems, Exponential Asymptotic Stability, Global Stablity, Poincaré Map

\section{Introduction}

Filippov introduces a solution concept for differential equations with discontinuous right-hand side [2]. Such equations frequently appear in economic modelling [6], [3], [1], [4], [5]. Despite the importance of these models regarding economic policy analysis and implications, economic analysis, however,

${ }^{1}$ EPSRC Research Grant (Engineering and Physical Science Research Council, 20112016), 1091684, Stability in Nonsmooth Systems with Applications to Biomechanics 
rarely goes beyond existence and uniqueness results. In this paper, we consider an example of a planar dynamical system defined by a set of autonomous ordinary differential equations with discontinuous right-hand side, and show how to calculate its solution. The solution is then used to establish global stability of a nonsmooth periodic orbit via Poincaré map. A property of the stability theory discussed here is that it requires calculating the explicit solution of the nonsmooth dynamical system, and then, by application of Poincaré theory to show that it is globally stable. Poincaré theory heavily relies on the information provided by the periodic orbit. Such information might not be available in many complex economic applications. We are hence interested in a theory that would not require such explicit information about the solution of the system.

In this paper we compare Poincaré's global stability theory to a local stability theory introduced in Stiefenhofer and Giesl [8], which does not require the calculation of a solution of the system at hand. At the case of an example we compare the two theories. In this paper, we discuss Poincaré's stability and derive the nonsmooth periodic orbit. We revisit this example in a companion paper of this journal and show that exponentially asymptotically stability can be shown without the explicit solution of a system.

The next section considers an example and derives the nonsmooth periodic orbit of a system with discontinuous right-hand side. Section three defines a Poincaré map and shows stability of the nonsmooth orbit. Section four is a conclusion.

\section{The model and its solution}

We consider an explicit calculation of a periodic orbit of a non-smooth dynamical system (1) defined by equations (2) and (3). The calculation of a periodic orbit is performed by transforming the original problem into a problem in polar coordinates. The explicit solution is then found by separation of variables. We show stability of this orbit by application of a Poincaré map.

We consider a differential equation

$$
\dot{x}=f(x),
$$

where $f$ is a discontinuous function at $x_{2}=0$ and $x \in \mathbb{R}^{2}$ such that for $f:=f^{ \pm}$ we have

$$
\dot{x}=f^{ \pm}(x)= \begin{cases}f^{+}(x) & \text { if } x_{2}>0 \\ f^{-}(x) & \text { if } x_{2}<0\end{cases}
$$

Let equation (1) be defined by 


$$
\left.\begin{array}{l}
f_{1}^{+}(x)=x_{1}\left(\left(R^{+}\right)^{2}-\left(\left(x_{1}\right)^{2}+\left(x_{2}\right)^{2}\right)\right)-x_{2} \\
f_{2}^{+}(x)=x_{2}\left(\left(R^{+}\right)^{2}-\left(\left(x_{1}\right)^{2}+\left(x_{2}\right)^{2}\right)\right)+x_{1}
\end{array}\right\} \text { if } x_{2}>0
$$

and

$$
\left.\begin{array}{l}
f_{1}^{-}(x)=x_{1}\left(\left(R^{-}\right)^{2}-\left(\left(x_{1}\right)^{2}+\left(x_{2}\right)^{2}\right)\right)-x_{2} \\
f_{2}^{-}(x)=x_{2}\left(\left(R^{-}\right)^{2}-\left(\left(x_{1}\right)^{2}+\left(x_{2}\right)^{2}\right)\right)+x_{1}
\end{array}\right\} \text { if } x_{2}<0
$$

where $R^{ \pm}>0$ are some constants [7].

The aim here is to calculate a solution of equations (2) and (3) via conventional theory. We also show that the periodic orbit is globally stable. This requires to define a Poincaré map. We want to find a solution of this model. Hence we transform the model in polar coordinates. We use the formulas

$$
\begin{aligned}
\dot{r}^{ \pm} & =\frac{1}{r^{ \pm}}\left(x_{1}^{ \pm} \dot{x}_{1}^{ \pm}+x_{2}^{ \pm} \dot{x}_{2}^{ \pm}\right) \\
\dot{\theta}^{ \pm} & =\frac{x_{1}^{ \pm} \dot{x}_{2}^{ \pm}+x_{2} \dot{x}_{1}^{ \pm}}{\left(r^{ \pm}\right)^{2}} .
\end{aligned}
$$

Using equations (4) and (5) we obtain

$$
\begin{aligned}
& \dot{r}^{ \pm}=r^{ \pm}\left(\left(R^{ \pm}\right)^{2}-\left(r^{ \pm}\right)^{2}\right) \\
& \dot{\theta}^{ \pm}=1 .
\end{aligned}
$$

The differential equation of the phase path is given by

$$
\frac{\frac{d r^{ \pm}}{d t}}{\frac{d \theta^{ \pm}}{d t}}=\frac{d r^{ \pm}}{d \theta^{ \pm}}=\frac{r^{ \pm}\left(\left(R^{ \pm}\right)^{2}-\left(r^{ \pm}\right)^{2}\right)}{1}
$$

This differential equation can be solved by the method of separating variables. Thus separating variables and integrating both sides yields

$$
\int \frac{d r^{ \pm}}{r^{ \pm}\left(\left(R^{ \pm}\right)^{2}-\left(r^{ \pm}\right)^{2}\right)}=\int \frac{d \theta^{ \pm}}{1}
$$

In order to find a solution of the l.h.s of equation (8) we apply partial sums. Hence, we have

$$
\int \frac{1}{r^{ \pm}\left(R^{ \pm}-r^{ \pm}\right)\left(R^{ \pm}+r^{ \pm}\right)} d r^{ \pm}=\int \frac{A}{r^{ \pm}} d r^{ \pm}+\int \frac{B}{\left(R^{ \pm}-r^{ \pm}\right)} d r^{ \pm}+\int \frac{C}{\left(R^{ \pm}+r^{ \pm}\right)} d r^{ \pm} .
$$

We solve

$$
1=A^{ \pm}\left(R^{ \pm}-r^{ \pm}\right)\left(R^{ \pm}+r^{ \pm}\right)+B^{ \pm} r^{ \pm}\left(R^{ \pm}+r^{ \pm}\right)+C^{ \pm} r^{ \pm}\left(R^{ \pm}-r^{ \pm}\right) .
$$


in order to obtain values for the constants A,B, and C. Hence equation (10) with $r^{ \pm}=0$ yields $A^{ \pm}=\frac{1}{\left(R^{ \pm}\right)^{2}}$. Equation (10) with $r^{ \pm}=R^{ \pm}$yields $B^{ \pm}=$ $\frac{1}{2\left(R^{ \pm}\right)^{2}}$, and with $r^{ \pm}=-R^{ \pm}$yields $C^{ \pm}=-\frac{1}{2\left(R^{ \pm}\right)^{2}}$. Substituting these constants back into equation (9) yields

$$
\begin{aligned}
& =\int \frac{\frac{1}{\left(R^{ \pm}\right)^{2}}}{r^{ \pm}} d r^{ \pm}+\int \frac{\frac{1}{2\left(R^{ \pm}\right)^{2}}}{\left(R^{ \pm}-r^{ \pm}\right)} d r^{ \pm}+\int \frac{-\frac{1}{2\left(R^{ \pm}\right)^{2}}}{\left(R^{ \pm}+r^{ \pm}\right)} d r^{ \pm} \\
& =\int \frac{1}{\left(R^{ \pm}\right)^{2} r^{ \pm}} d r^{ \pm}+\int \frac{1}{2\left(R^{ \pm}\right)^{2}\left(R^{ \pm}-r^{ \pm}\right)} d r^{ \pm}-\int \frac{1}{2\left(R^{ \pm}\right)^{2}\left(R^{ \pm}+r^{ \pm}\right)} d r^{ \pm} \\
& =\frac{1}{\left(R^{ \pm}\right)^{2}} \int \frac{1}{r^{ \pm}} d r^{ \pm}+\frac{1}{2\left(R^{ \pm}\right)^{2}} \int \frac{1}{\left(R^{ \pm}-r^{ \pm}\right)} d r^{ \pm}-\frac{1}{2\left(R^{ \pm}\right)^{2}} \int \frac{1}{\left(R^{ \pm}+r^{ \pm}\right)} d r^{ \pm}
\end{aligned}
$$

Now solving equation (8) yields

$$
\begin{aligned}
\frac{1}{\left(R^{ \pm}\right)^{2}} \ln r^{ \pm}-\frac{1}{2\left(R^{ \pm}\right)^{2}} \ln \left(R^{ \pm}-r^{ \pm}\right)-\frac{1}{2\left(R^{ \pm}\right)^{2}} \ln \left(R^{ \pm}+r^{ \pm}\right) & =\theta^{ \pm}+c_{1}^{ \pm} \\
\frac{1}{2\left(R^{ \pm}\right)^{2}} \ln r^{ \pm}+\frac{1}{2\left(R^{ \pm}\right)^{2}} \ln r^{ \pm}-\frac{1}{2\left(R^{ \pm}\right)^{2}} \ln \left(R^{ \pm}-r^{ \pm}\right)-\frac{1}{2\left(R^{ \pm}\right)^{2}} \ln \left(R^{ \pm}+r^{ \pm}\right) & =\theta^{ \pm}+c_{1}^{ \pm} \\
\frac{1}{2\left(R^{ \pm}\right)^{2}} \ln \left(\frac{\left(r^{ \pm}\right)^{2}}{\left(R^{ \pm}-r^{ \pm}\right)\left(R^{ \pm}+r^{ \pm}\right)}\right) & =\theta^{ \pm}+c_{1}^{ \pm} . \\
\left(\frac{\left(r^{ \pm}\right)^{2}}{\left(R^{ \pm}-r^{ \pm}\right)\left(R^{ \pm}+r^{ \pm}\right)}\right) & =e^{2\left(R^{ \pm}\right)^{2}\left(\theta^{ \pm}+c_{1}^{ \pm}\right)} .
\end{aligned}
$$

Hence we have

$$
\left(\frac{\left(r^{ \pm}\right)^{2}}{\left(R^{ \pm}-r^{ \pm}\right)\left(R^{ \pm}+r^{ \pm}\right)}\right)=e^{2\left(R^{ \pm}\right)^{2}\left(\theta^{ \pm}+c_{1}^{ \pm}\right)}
$$

with $D^{ \pm}=e^{c^{ \pm}}, r_{0}^{ \pm}=r_{0}^{ \pm}\left(\theta_{0}^{ \pm}\right)$, and $\theta_{0}^{ \pm}$we obtain

$$
D^{ \pm}=\frac{\left(r_{0}^{ \pm}\right)^{2}}{\left(\left(R^{ \pm}\right)^{2}-\left(r_{0}^{ \pm}\right)^{2}\right)} e^{-2 \theta_{0}^{ \pm}\left(R^{ \pm}\right)^{2}}
$$

Substituting equation (12) into equation (11) yields

$$
\begin{aligned}
\left(\frac{\left(r^{ \pm}\right)^{2}}{\left(R^{ \pm}-r^{ \pm}\right)\left(R^{ \pm}+r^{ \pm}\right)}\right) & =e^{\left.2 \theta^{ \pm} R^{ \pm}\right)^{2}} D^{ \pm} \\
\left(\frac{\left(r^{ \pm}\right)^{2}}{\left(\left(R^{ \pm}\right)^{2}-\left(r^{ \pm}\right)^{2}\right)}\right) & =\frac{\left(r_{0}^{ \pm}\right)^{2}}{\left(\left(R^{ \pm}\right)^{2}-\left(r_{0}^{ \pm}\right)^{2}\right)} e^{-2\left(\theta_{0}^{ \pm}-\theta^{ \pm}\right)\left(R^{ \pm}\right)^{2}}
\end{aligned}
$$

which after some algebra yields

$$
\left(r^{ \pm}\right)^{2}=\frac{\left(\frac{\left(r_{0}^{ \pm}\right)^{2}}{\left(\left(R^{ \pm}\right)^{2}-\left(r^{ \pm}\right)^{2}\right)} e^{-2 \theta_{0}^{ \pm}\left(R^{ \pm}\right)^{2}}\left(R^{ \pm}\right)^{2}\right) e^{2 \theta^{ \pm}\left(R^{ \pm}\right)^{2}}}{1+\left(\frac{\left(r_{0}^{ \pm}\right)^{2}}{\left(\left(R^{ \pm}\right)^{2}-\left(r^{ \pm}\right)^{2}\right)} e^{-2 \theta_{0}^{ \pm}\left(R^{ \pm}\right)^{2}}\right) e^{2 \theta^{ \pm}\left(R^{ \pm}\right)^{2}}}
$$


and simplifies to

$$
\left(r^{ \pm}\right)^{2}=\frac{\left(R^{ \pm}\right)^{2}}{e^{-2\left(\theta^{ \pm}-\theta_{0}^{ \pm}\right)\left(R^{ \pm}\right)^{2}}\left(\frac{\left(R^{ \pm}\right)^{2}-\left(r_{0}^{ \pm}\right)^{2}}{\left(r_{0}^{ \pm}\right)^{2}}\right)+1} .
$$

Now we consider the positive orbit. Hence let $\theta_{0}^{+}=0$, and $\theta_{1}^{+}=\pi$, then

$$
\left(r_{1}^{+}\right)^{2}=\frac{\left(R^{+}\right)^{2}}{e^{-2 \pi\left(R^{+}\right)^{2}}\left(\frac{\left(R^{+}\right)^{2}-\left(r_{0}^{+}\right)^{2}}{\left(r_{0}^{+}\right)^{2}}\right)+1} .
$$

Let's consider the negative orbit. Hence let $\theta_{1}^{-}=\pi$, and $\theta_{2}^{-}=2 \pi$, then

$$
\left(r_{2}^{-}\right)^{2}=\frac{\left(R^{-}\right)^{2}}{e^{-2 \pi\left(R^{-}\right)^{2}}\left(\frac{\left(R^{-}\right)^{2}-\left(r_{1}^{-}\right)^{2}}{\left(r_{1}^{-}\right)^{2}}\right)+1} .
$$

We now define the Poincaré map

$$
P^{2}:\left(r_{0}^{+}\right)^{2} \rightarrow\left(r_{1}^{+}\right)^{2}=\left(r_{1}^{-}\right)^{2} \rightarrow\left(r_{2}^{-}\right)^{2}
$$

using equations (14) and (15) equation by

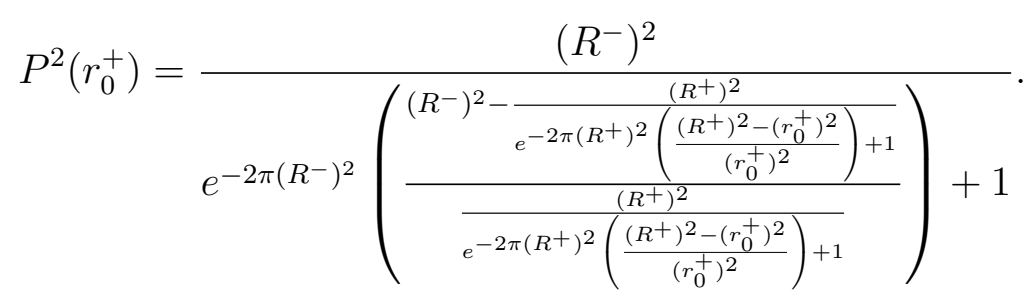

We want to find a fixed point

$$
P^{2}\left(r_{0}^{+}\right)=\left(r_{0}^{+}\right)^{2}
$$

and show that

$$
\left|P^{\prime}\left(\rho_{0}^{i}\right)\right|<1 .
$$

with $\rho_{i}:=\left(r_{i}\right)^{2}$ and $i=0,1$. Now, we calculate

$$
\bar{P}^{2}\left(r_{0}^{+}\right)=\left(r_{0}^{+}\right)^{2}
$$

using equation (14). We have by equation (14)

$$
\begin{aligned}
\left(r_{1}^{+}\right)^{2} & =\frac{\left(R^{+}\right)^{2}}{e^{-2 \pi\left(R^{+}\right)^{2}}\left(\frac{\left(R^{+}\right)^{2}-\left(r_{0}^{+}\right)^{2}}{\left(r_{0}^{+}\right)^{2}}\right)+1} \\
& =\frac{A^{2}}{\frac{B^{2}-\left(r_{0}^{+}\right)^{2}}{\left(r_{0}^{+}\right)^{2}}+\frac{A^{2}}{B^{2}}}
\end{aligned}
$$


with

$$
\begin{aligned}
& A^{2}=e^{2 \pi\left(R^{+}\right)^{2}\left(R^{+}\right)^{2}} \\
& B^{2}=\left(R^{+}\right)^{2} .
\end{aligned}
$$

Then using (17) we obtain

$$
\begin{aligned}
\frac{A^{2}\left(r_{0}^{+}\right)^{2}}{B^{2}-\left(r_{0}^{+}\right)^{2}+\frac{A^{2}}{B^{2}}\left(r_{0}^{+}\right)^{2}} & =\left(r_{0}^{+}\right)^{2} \\
A^{2}\left(r_{0}^{+}\right)^{2} & =\left(r_{0}^{+}\right)^{2}\left[B^{2}-\left(r_{0}^{+}\right)^{2}+\frac{A^{2}}{B^{2}}\left(r_{0}^{+}\right)^{2}\right],
\end{aligned}
$$

hence

$$
r_{0}^{+}=0
$$

is a solution. Other solutions are given by

$$
\begin{aligned}
A^{2} & =\left[B^{2}-\left(r_{0}^{+}\right)^{2}+\frac{A^{2}}{B^{2}}\left(r_{0}^{+}\right)^{2}\right] \\
A^{2}-B^{2} & =\left(r_{0}^{+}\right)^{2}\left[\frac{A^{2}}{B^{2}}-1\right] \\
\left(r_{0}^{+}\right)^{2} & =\frac{A^{2}-B^{2}}{\frac{A^{2}}{B^{2}}-\frac{B^{2}}{B^{2}}} \\
& =\frac{A^{2}-B^{2}}{A^{2}-B^{2}} B^{2}
\end{aligned}
$$

which by substitution of $B^{2}=\left(R^{+}\right)^{2}$ and simplification yields the solution

$$
r_{0}^{+}=\sqrt{\left(R^{+}\right)^{2}}=R^{+} .
$$

Now, we calculate

$$
P^{2}\left(r_{0}^{+}\right)=\left(r_{0}^{+}\right)^{2}
$$

using equation (16). We have by equation (16)

$$
\begin{aligned}
\left(r_{2}^{-}\right)^{2} & =\frac{\left(R^{-}\right)^{2}}{e^{-2 \pi\left(R^{-}\right)^{2}}\left(\frac{\left(R^{-}\right)^{2}-\left(r_{1}^{-}\right)^{2}}{\left(r_{1}^{-}\right)^{2}}\right)+1} \\
& =\frac{a^{2}}{\frac{b^{2}-\left(r_{1}^{-}\right)^{2}}{\left(r_{1}^{-}\right)^{2}}+\frac{a^{2}}{b^{2}}}
\end{aligned}
$$


with

$$
\begin{aligned}
& a^{2}=e^{2 \pi\left(R^{-}\right)^{2}}\left(R^{-}\right)^{2} \\
& b^{2}=\left(R^{-}\right)^{2}
\end{aligned}
$$

Then using (18) we obtain

$$
\begin{aligned}
& \frac{a^{2}\left(r_{1}^{-}\right)^{2}}{b^{2}-\left(r_{1}^{-}\right)^{2}+\frac{a^{2}}{b^{2}}\left(r_{1}^{-}\right)^{2}}=\left(r_{0}^{+}\right)^{2} \\
& a^{2}\left(r_{1}^{-}\right)^{2}=\left(r_{0}^{+}\right)^{2}\left[b^{2}-\left(r_{1}^{-}\right)^{2}+\frac{a^{2}}{b^{2}}\left(r_{1}^{-}\right)^{2}\right] \\
& \frac{a^{2}}{\left(r_{0}^{+}\right)^{2}}\left[\frac{B^{2}-\left(r_{0}^{+}\right)^{2}}{\left(r_{0}^{+}\right)^{2}}+\frac{A^{2}}{B^{2}}\right]=\left[b^{2}+\left(\frac{a^{2}}{b^{2}}-1\right)\right]\left[\frac{B^{2}-\left(r_{0}^{+}\right)^{2}}{\left(r_{0}^{+}\right)^{2}}+\frac{A^{2}}{B^{2}}\right] \\
& \frac{a^{2}}{\left(r_{0}^{+}\right)^{2}}=\frac{b^{2}\left(r_{0}^{+}\right)^{2}}{\frac{A^{2}\left(r_{0}^{+}\right)^{2}}{B^{2}-\left(r_{0}^{+}\right)^{2}+\frac{A^{2}}{B^{2}}\left(r_{0}^{+}\right)^{2}}}+\frac{a^{2}-b^{2}}{b^{2}} \\
& =\frac{b^{2}\left[B^{2}-\left(r_{0}^{+}\right)^{2}+\frac{A^{2}}{B^{2}}\left(r_{0}^{+}\right)^{2}\right]}{A^{2}\left(r_{0}^{+}\right)^{2}}+\frac{a^{2}-b^{2}}{b^{2}} \\
& \frac{a^{2}-b^{2}}{b^{2}}=\frac{1}{\left(r_{0}^{+}\right)^{2}}\left[a^{2}-\frac{b^{2} B^{2}-b^{2}\left(r_{0}^{+}\right)^{2}+\frac{A^{2}}{B^{2}} b^{2}\left(r_{0}^{+}\right)^{2}}{A^{2}}\right] \\
& \left(\frac{a^{2}-b^{2}}{b^{2}}\right)\left(r_{0}^{+}\right)^{2}=\left(r_{0}^{+}\right)^{2}\left(b^{2}-\frac{A^{2}}{B^{2}} b^{2}\right)+A^{2} a^{2}-b^{2} B^{2} \\
& A^{2} a^{2}-b^{2} B^{2}=\left(r_{0}^{+}\right)^{2}\left(A^{2}\left[\frac{a^{2}-b^{2}}{b^{2}}\right]+b^{2}\left[\frac{A^{2}-B^{2}}{B^{2}}\right]\right) \\
& \left(r_{0}^{+}\right)^{2}=\frac{A^{2} a^{2}-b^{2} B^{2}}{\left(A^{2}\left[\frac{a^{2}-b^{2}}{b^{2}}\right]+b^{2}\left[\frac{A^{2}-B^{2}}{B^{2}}\right]\right)}
\end{aligned}
$$

which after substitution of $A, B, a, b$ yields

$$
\begin{aligned}
\left(r_{0}^{+}\right)^{2} & =\frac{e^{2 \pi\left(R^{+}\right)^{2}}\left(R^{+}\right)^{2} e^{2 \pi\left(R^{-}\right)^{2}}\left(R^{-}\right)^{2}-\left(R^{-}\right)^{2}\left(R^{+}\right)^{2}}{\left(e^{2 \pi\left(R^{+}\right)^{2}}\left(R^{+}\right)^{2}\left[\frac{e^{2 \pi\left(R^{-}\right)^{2}\left(R^{-}\right)^{2}-\left(R^{-}\right)^{2}}}{\left(R^{-}\right)^{2}}\right]+\left(R^{-}\right)^{2}\left[\frac{e^{2 \pi\left(R^{+}\right)^{2}\left(R^{+}\right)^{2}-\left(R^{+}\right)^{2}}}{\left(R^{+}\right)^{2}}\right]\right)} \\
& =\frac{\left(R^{+}\right)^{2}\left(R^{-}\right)^{2}\left[e^{2 \pi\left(\left(R^{+}\right)^{2}\left(R^{-}\right)^{2}\right)}-1\right]}{\left(R^{+}\right)^{2}\left(R^{-}\right)^{2}\left[\frac{e^{2 \pi\left(\left(R^{+}\right)^{2}\left(R^{-}\right)^{2}\right)}}{\left(R^{-}\right)^{2}}-\frac{e^{2 \pi\left(R^{-}\right)^{2}}}{\left(R^{-}\right)^{2}}+\frac{e^{2 \pi\left(R^{+}\right)^{2}}}{\left(R^{+}\right)^{2}}-\frac{1}{\left(R^{+}\right)^{2}}\right]} \\
& =\frac{\left(R^{+}\right)^{2}\left(R^{-}\right)^{2}\left[e^{2 \pi\left(\left(R^{+}\right)^{2}\left(R^{-}\right)^{2}\right)}-1\right]}{\left(e^{2 \pi\left(R^{+}\right)^{2}}-1\right)\left(e^{2 \pi\left(R^{-}\right)^{2}}\left(R^{+}\right)^{2}+\left(R^{-}\right)^{2}\right)}
\end{aligned}
$$


Hence we have the solution

$$
r_{0}^{+}=\sqrt{\frac{\left(R^{+}\right)^{2}\left(R^{-}\right)^{2}\left[e^{2 \pi\left(\left(R^{+}\right)^{2}\left(R^{-}\right)^{2}\right)}-1\right]}{\left(e^{2 \pi\left(R^{+}\right)^{2}}-1\right)\left(e^{2 \pi\left(R^{-}\right)^{2}}\left(R^{+}\right)^{2}+\left(R^{-}\right)^{2}\right)}} .
$$

\section{Global stability of the nonsmooth periodic orbit}

We now want to show stability of the periodic orbit. This requires to check that the absolute value of the derivative of the Poincaré map is less than one at the calculated fixed point.

Let the radius $r$ go from initial value $r_{0}$ to $r_{1}$ and then to $r_{2}$, and let $\rho_{i}=r_{i}^{2}$. It is enough to show that $\Pi\left(\rho_{0}\right)=P^{2}\left(r_{0}^{2}\right)$ satisfies $\left|\Pi^{\prime}\left(\rho_{0}\right)\right|<1$ (so everything for the squares): Indeed, we have

$$
\begin{aligned}
\frac{d r_{2}}{d r_{0}} & =\frac{d \sqrt{\rho_{2}}}{d \rho_{0}} \frac{d \rho_{0}}{d r_{0}} \\
& =\frac{1}{2} \rho_{2}^{-1 / 2} \frac{d \rho_{2}}{d \rho_{0}} 2 r_{0} \\
& =\frac{d \rho_{2}}{d \rho_{0}} \frac{r_{0}}{r_{2}}
\end{aligned}
$$

Note that at the fixed point (periodic orbit) we have $r_{0}=r_{2}$, so that $\frac{d r_{2}}{d r_{0}}=\frac{d \rho_{2}}{d \rho_{0}}$.

Now let's calculate $\frac{d \rho_{2}}{d \rho_{0}}$. We have

$$
\begin{aligned}
& \rho_{1}=\frac{\left(R^{+}\right)^{2} e^{2 \pi\left(R^{+}\right)^{2}}}{\frac{\left(R^{+}\right)^{2}}{\rho_{0}}-1+e^{2 \pi\left(R^{+}\right)^{2}}}, \\
& \rho_{2}=\frac{\left(R^{-}\right)^{2} e^{2 \pi\left(R^{-}\right)^{2}}}{\frac{\left(R^{-}\right)^{2}}{\rho_{1}}-1+e^{2 \pi\left(R^{-}\right)^{2}}} .
\end{aligned}
$$

In particular we have from the first equation

$$
\rho_{1}\left(\frac{\left(R^{+}\right)^{2}}{\rho_{0}}-1+e^{2 \pi\left(R^{+}\right)^{2}}\right)=\left(R^{+}\right)^{2} e^{2 \pi\left(R^{+}\right)^{2}}
$$


Then

$$
\begin{aligned}
\Pi^{\prime}\left(\rho_{0}\right)= & \frac{d \rho_{2}}{d \rho_{1}} \frac{d \rho_{1}}{d \rho_{0}} \\
= & \frac{\left(R^{-}\right)^{2} e^{2 \pi\left(R^{-}\right)^{2}}}{\left(\frac{\left(R^{-}\right)^{2}}{\rho_{1}}-1+e^{2 \pi\left(R^{-}\right)^{2}}\right)^{2}} \frac{\left(R^{-}\right)^{2}}{\rho_{1}^{2}} \\
& \times \frac{\left(R^{+}\right)^{2} e^{2 \pi\left(R^{+}\right)^{2}}}{\left(\frac{\left(R^{+}\right)^{2}}{\rho_{0}}-1+e^{2 \pi\left(R^{+}\right)^{2}}\right)^{2}} \frac{\left(R^{+}\right)^{2}}{\rho_{0}^{2}} \\
= & \frac{\left(R^{-}\right)^{4} e^{2 \pi\left(R^{-}\right)^{2}}\left(R^{+}\right)^{4} e^{2 \pi\left(R^{+}\right)^{2}}}{\left(\frac{\left(R^{-}\right)^{2}}{\rho_{1}}-1+e^{2 \pi\left(R^{-}\right)^{2}}\right)^{2}\left(R^{+}\right)^{4} e^{4 \pi\left(R^{+}\right)^{2} \rho_{0}^{2}}} \text { using } \\
= & \frac{\left(R^{-}\right)^{4} e^{2 \pi\left(R^{-}\right)^{2}} e^{2 \pi\left(R^{+}\right)^{2}}}{\left(\frac{\left(R^{-}\right)^{2}}{\rho_{1}}-1+e^{2 \pi\left(R^{-}\right)^{2}}\right)^{2} \rho_{0}^{2} e^{4 \pi\left(R^{+}\right)^{2}}} .
\end{aligned}
$$

The fixed point (periodic orbit) condition is $\rho_{2}=\rho_{0}$, i.e. see second equation (19)

$$
\begin{aligned}
\rho_{0} & =\frac{\left(R^{-}\right)^{2} e^{2 \pi\left(R^{-}\right)^{2}}}{\frac{\left(R^{-}\right)^{2}}{\rho_{1}}-1+e^{2 \pi\left(R^{-}\right)^{2}}} \\
\left(\frac{\left(R^{-}\right)^{2}}{\rho_{1}}-1+e^{2 \pi\left(R^{-}\right)^{2}}\right)^{2} \rho_{0}^{2} & =\left(R^{-}\right)^{4} e^{4 \pi\left(R^{-}\right)^{2}}
\end{aligned}
$$

This gives

$$
\begin{aligned}
\Pi^{\prime}\left(\rho_{0}\right) & =\frac{e^{2 \pi\left(R^{-}\right)^{2}} e^{2 \pi\left(R^{+}\right)^{2}}}{e^{4 \pi\left(R^{-}\right)^{2} e^{4 \pi\left(R^{+}\right)^{2}}}} \\
& =e^{-2 \pi\left(\left(R^{-}\right)^{2}+\left(R^{+}\right)^{2}\right)}<1
\end{aligned}
$$

By inspection of this inequality we conclude that the periodic orbit is stable.

\section{Conclusion}

In this paper we consider a system of ordinary differential equations with discontinuous right-hand side. At the case of an example, we explicitly calculate the nonsmooth periodic orbit of such a dynamical system and show by application of a Poincaré map that it is globally stable. The main drawback of this theory, however, is that it requires the explicit calculation of the solution of the system at hand. This may be demanding and in some economic 
applications not even always possible. This problem may prevent economists from deriving analytic solutions of more complex economic problems for the purpose of economic policy analysis. A companion paper revisits the example considered here, and shows that Stiefenhofer and Giesl [8] provide a local theory of exponentially asymptotically stability of nonsmooth periodic orbits and a formula for the basin of attraction, which does not require the calculation of the solution of the dynamical system.

\section{References}

[1] J. P. Benassy, A neo-Keynesian model of price and quantity determination in disequilibrium, in: ed. G. Schwödiauer, editor, Equilibrium and disequilibrium in economic theory, Reidel, Dordrecht, 1978, 511-544. https://doi.org/10.1007/978-94-010-1155-6_27

[2] A. F. Filippov, Differential Equations with Discontinuous Righthand Sides, Kluwier Academic Press, 1988. https://doi.org/10.1007/978-94015-7793-9

[3] C. Henry, Differential equations with discontinuous right-hand side for palning procedure, Journal of Economic Theory, 4 (1972), 545-551.

https://doi.org/10.1016/0022-0531(72)90138-x

[4] T. Ito, A Filippov solution of a system of differential equations with discontinuous right-hand side, Economic Letters, 4 (1979), 349-354.

https://doi.org/10.1016/0165-1765(79)90183-6

[5] K. G. Löfgren, The corridor and local stability of the effective excess demand hypothesis: A result, Scandinavian Journal of Economics, 81 (1979), 40-47. https://doi.org/10.2307/3439455

[6] F. Mallivaud, The theory of unemployment reconsidered, Basil Blackwell, Oxford, 1977.

[7] P. Stiefenhofer, Stability analysis of non-smooth dynamical systems with an application to biomechanics, University of Sussex, School of Mathematics and Physical Sciences, 2016.

[8] P. Stiefenhofer and P. Giesl, Economic periodic orbits: a theory of asymptotic stability, Nonlinear Analysis and Differential Equations, 7(1) (2019), 9-16. https://doi.org/10.12988/nade.2019.923

\section{Received: April 25, 2019; Published: May 26, 2019}

